UCRL-JC-128575

PREPRINT

\title{
Steering Algorithms for a Small Recirculating Heavy-Ion Accelerator
}

\author{
W. M. Sharp \\ D. P. Grote \\ M. A. Hernandez \\ G. W. Kamin
}

This paper was prepared for submittal to the 12th International Symposium on Heavy-Ion Inertial Fusion

Heidelberg, Germany

September 23-27, 1997

November 7, 1997

This is a preprint of a paper intended for publication in a journal or proceedings. Since changes may be made before publication, this preprint is made available with the understanding that it will not be cited or reproduced without the permission of the author. 


\title{
Steering Algorithms for a Small Recirculating Heavy-Ion Accelerator*
}

\author{
W. M. Sharp, D. P. Grote, M. A. Hernandez, and G. W. Kamin \\ Lawrence Livermore National Laboratory, University of California \\ Livermore, California 94550, USA
}

\begin{abstract}
Beam-steering algorithms are proposed for a small recirculating induction accelerator being built at the Lawrence Livermore National Laboratory. The principal problem is that the transverse position and velocity of the beam must be inferred from capacitive position monitors, and this determination is complicated by the limited probe resolution and by the lattice errors within steering modules. The fluid/envelope code CIRCE is used to evaluate these algorithms.
\end{abstract}

\section{Introduction}

A recirculating heavy-ion induction accelerator $[1,2]$ has been suggested as an alternative to conventional linear drivers for heavy-ion fusion (HIF). This "recirculator" is expected to cost significantly less than linear drivers because the accelerating cells are used repeatedly for each pulse and because smaller induction units with a reduced accelerating gradient would be used.

The circular design of such machines requires the use of dipoles with time-varying field strengths to bend to a beam along the design trajectory. If the strength of any dipole is inappropriate for the beam momentum, due to improper timing or to an erroneous drive voltage, then the beam will, in effect, receive a small transverse kick as it traverses the dipole. Transverse misalignments of quadrupoles and any rotation of dipoles about the beam axis similarly give transverse kicks to the beam centroid. The main effect of these transverse kicks is that the amplitude of the beam-centroid betatron motion increases, and if the kicks are uncorrelated, then the increase should resemble a random walk. [3] A second effect occurs in an intense ion pulse because image forces cause the betatron frequency to decrease along the beam by an amount roughly proportional to the current. When the beam is displaced from the design trajectory due to centroid kicks, the difference in betatron frequency along the beam leads to an increasing slippage in betatron phase between the beam ends and the center, which shows up as centroid ripples along the beam with axial wavelengths that become shorter with time. Eventually field nonlinearities and axial slippage in bends are expected to thermalize these centroid ripples, leading to emittance growth near the ends. Both the increasing amplitude of betatron motion and the emittance growth due to rippling are undesirable in a HIF driver because they greatly complicate the final focusing of the beam onto the target.

* Work performed under the auspices of the U. S. Department of Energy by Lawrence Livermore National Laboratory under contract W-7405-ENG-48. 
A series of scaled experiments to study the physics and engineering issues of recirculators is being carried out at the Lawrence Livermore National Laboratory (LLNL). [2] Despite the small size and the low current and energy of the accelerator, these experiments pose the same problem of controlling the transverse beam position that is expected in a recirculating HIF driver. To allow realistic error tolerances on the strength and alignment of lattice elements, the experimental beam must be steered repeatedly using only transverseposition data from capacitive probes. [4] However, the limited probe resolution and the lattice errors within steering modules complicate the calculation of correction signals. In this paper, simple steering algorithms are proposed for correcting centroid errors, and these algorithms and some refinements are assessed using the fluid/envelope code CIRCE. [5]

\section{Steering Algorithm}

The strategy discussed here for reducing centroid displacements due to reproducible lattice errors involves sensing centroid errors of one pulse and correcting them in subsequent pulses. The high shot-to-shot repeatability seen in induction accelerators makes this approach feasible. All informations about centroid trajectories is inferred from beam-position monitors, and errors in the centroid position and velocity are corrected by finite-length steering dipoles. In general, different algorithms are needed for the two transverse directions due to the effect of bend fields in the plane of the accelerator, but since centroid coordinate $X$ in the accelerator plane is uncoupled to lowest order from coordinate $Y$ in the perpendicular direction when alternating-gradient focusing is used, steering corrections may by calculated independently in the two directions.

\subsection{Steering in the accelerator plane}

Errors in the centroid transverse position $X$ and velocity $X^{\prime} \equiv d X / d s$ in the accelerator plane are corrected in steering modules with at least five steering elements. Two dipoles are needed for steering, and the transverse position must be measured at a minimum of three monitors, since the centroid trajectory in a bend depends on the momentum error $\Delta p / p=1-p_{0} / p$, as well as on the initial values of $X$ and $X^{\prime}$. Here, $p=\gamma \beta M c$ is the actual momentum of an ion with mass $M$, and $p_{0}=F_{x} \rho /(\beta c)$ is the design momentum for a dipole with bend radius of $\rho$ and a nominal bending force $F_{x}$, given by either $q e E_{x}$ for electric dipoles or $q e \beta c B_{y}$ for magnetic dipoles. In these expressions for $p$ and $p_{0}, \beta$ is the beam longitudinal velocity scaled by the speed of light $c$, and $\gamma=\left(1-\beta^{2}\right)^{-\frac{1}{2}}$ is the Lorentz factor.

Briefly, the steering algorithm uses the linear transport matrices representing the idealized lattice to estimate $X, X^{\prime}$, and $\Delta p / p$ through the steering module, consistent with the beam-position data. The same matrices are used also to calculate matched values of $X$ and $X^{\prime}$ at the second steering dipole. The needed steering corrections are then calculated using a "thin-lens" approximation, modeling corrections as instantaneous changes in $X^{\prime}$ occurring at the midpoint of the steering dipoles. The kick at the first dipole is calculated to place the beam centroid at the matched $X$ position as it reaches the second steering dipole, and the kick there is chosen to change $X^{\prime}$ to the matched value. Fields for the actual finite-length dipoles that give the correct transverse impulse are then calculated 
under the assumption that the steering force and the beam position are approximately constant as a beam slice traverses the finite-length of the steering dipole. This thin-lens approximation is found to be valid so long as the dipole lengths are small compared with their separation.

Steering corrections may be either time dependent or independent of time on a single lap. For time-dependent steering, beam-position data are saved as functions of time, suitably averaged over time intervals to account for the finite monitor length. Appropriate kicks are then calculated for each of many closely spaced beam slices. In constrast, the kicks for time-independent steering are calculated for the center slice only and used for all slices. In either case, different corrections are in general required on each lap.

\subsection{Steering in the perpendicular direction}

In the direction perpendicular to the bend, the beam longitudinal momentum affects the trajectory only as small correction to the focusing and image forces, so the lowest-order trajectory is determined only by the initial values of $Y$ and $Y^{\prime} \equiv d Y / d s$. Consequently, the orbit through the steering module can be calculated with first-order transport theory using data from only two monitors, although the $\Delta p / p$ information from a third monitor could be used to obtain more accurate values for the beam rigidity and perveance in this orbit calculation. Alternately, redundant position data from a third monitor might be used to reduce the inaccuracies in the calculations arise from lattice errors between monitors. Once the values of $Y$ and $Y^{\prime}$ in the steering module have been estimated, the required transverse kicks at the two downstream steering dipoles are estimated just as for the $X$ corrections. The same two-monitor algorithm can, of course, be used also to steer in either transverse direction of a straight lattice section.

\subsection{Implementation in CIRCE}

The steering algorithm has been provisionally tested in the fast-running code CIRCE [5], which uses multiple envelope equations to model the transverse dynamics of beam slices and treats the longitudinal dynamics using a Lagrangian fluid model. The code has been benchmarked against the three-dimensional particle code WARP3d [6] and is found to be in good agreement so long as the longitudinal temperature is small and the beam transverse phase space remains single valued in all two-dimensional projections. These conditions are well satisfied in cases treated here, although the steering algorithm must still be verified using WARP3d to check for emittance growth due to steering and for any effects of fringe fields and multipoles in steering dipoles.

The basic steering algorithm is implemented in CIRCE in a particularly simple manner. To avoid redundant calculation, the multiple-shot procedure needed in an actual recirculator to set the steering dipoles is modeled in CIRCE by making two passes through each steering dipole during a single computer run. During the first pass of a pulse through a steering module, the centroid positions at the three monitors are saved as functions of time, and the steering corrections are calculated. The beam is then reinitialized at the first monitor, and the steering fields are applied during the second pass. 
Table 1 Nominal parameters of the LLNL small recirculator

$\begin{array}{lll}\text { beam parameters } & & \\ \text { ion charge state } & q & 1 \\ \text { ion mass } & M & 39 \mathrm{amu} \\ \text { initial beam current } & I_{b} & 2 \mathrm{~mA} \\ \text { initial energy } & \left(\gamma_{0}-1\right) M c^{2} & 80 \mathrm{MeV} \\ \text { initial duration } & \Delta \tau & 4 \mu \mathrm{s} \\ \text { initial rise/fall time } & \tau_{r} & 1 \mu \mathrm{s} \\ \text { lattice parameters } & & \\ \text { circumference } & s_{\max } & 14.4 \mathrm{~m} \\ \text { half-period } & L & 36 \mathrm{~cm} \\ \text { pipe radius } & R & 3.5 \mathrm{~cm} \\ \text { number of laps } & N & 15\end{array}$

\section{Assessment}

The steering algorithm is tested using the nominal lattice of the LLNL small recirculator. [2] Essential lattice parameters are listed in Table 1 along with the initial beam parameters. Each half-lattice period (HLP) consists of a focusing permanent-magnet quadrupole, a time-varying electric dipole for bending, and an acceleration gap. It is expected that both monitoring and steering of the beam centroid will be done by capacitive probes, referred to hereafter as "c-probes," placed around the beam-pipe inside each quadrupole. [4] Three half-lattice periods at longitudinal positions $s=0$ and at $s=\frac{1}{2} s_{\max }$, where $s_{\max }$ is the ring circumference, are reserved for beam, insertion and extraction, and neither acceleration nor steering are possible in these sections due to the complicated lattice geometry. The standard steering module here occupies five half-lattice periods, with three of the c-probes used as monitors (M) and the other two used as steering dipoles (S). The usual arrangement of monitors and steering dipoles is MSMSM, but the effectiveness of steering is found to depend only weakly on the order.

In the tests reported here, lattice errors of three types are introduced, all of which lead to transverse displacements of the centroid. Random errors are made in the field strengths and azimuthal alignment of bending dipoles, and in the transverse alignment of the focusing quadrupole magnets. For the dipoles, field-strength errors are taken to be the same fraction of the nominal strength on every lap, corresponding to bend voltages that are correctly timed but incorrect in magnitude. The dipole and quadrupole alignment errors are specified by the root mean-square (rms) values of the errors. In all cases, error magnitudes are arbitrarily chosen to have a uniform probability over a range chosen to give the appropriate rms error, although other error distributions, such as Gausssian, could equally be used. For each case presented here, the same set of lattice errors is used to allow a fair comparison of steering algorithms. A $0.2 \%$ rms error in bend strengths is used, and the alignment errors are $0.2^{\circ}$ for dipoles and $200 \mu \mathrm{m}$ in each transverse direction for quadrupoles. The dipole strength and alignment errors here are twice the specified experimental tolerances in order to guarantee that the steering algorithm works in the 

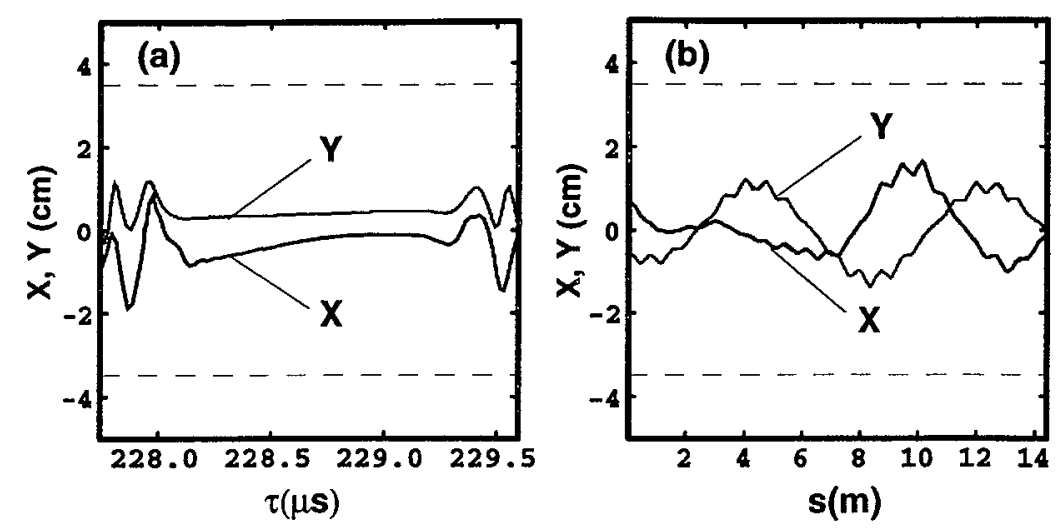

Fig. 1 Beam-centroid displacement with standard errors, shown by (a) a "snapshot" at the end of fifteen laps and (b) a "history" of the beam midpoint during the last lap. The dashed line marks the location of the beam-pipe wall.

worst likely cases.

The time-dependent and time-independent steering algorithms are tested separately here. However, both techniques would likely be used in a HIF driver, with between one and four time-dependent modules in a ring and a substantially larger number of timeindependent modules placed between them.

\subsection{Dynamics without steering}

To illustrate the need for steering in the small recirculator, CIRCE is first used to model an initially matched beam traversing the planned fifteen laps, using the standard set of errors but no steering. Two views of the resulting dynamics are shown in Fig. 1. The time variation of $X$ and $Y$ at the end of the last lap is shown in Fig. 1a, while Fig. 1b shows the centroid coordinates of the beam-midpoint during the final lap. The two types of plot are referred to hereafter respectively as "snapshots" and "histories." The snapshot in Fig. 1a shows the expected short-wavelength ripple along the beam due to the slippage of betatron phase between the ends and the center. This ripple is concentrated near the ends because the current, and therefore the betatron frequency, is relatively constant over the center half of the pulse. The history plot in Fig. 1b shows betatron oscillations in $X$ and $Y$ with amplitudes approaching $2 \mathrm{~cm}$. The short wavelength oscillations seen near the crests are the normal "flutter" motion of an off-axis beam traversing an alternatinggradient focusing system. The betatron amplitude here is sufficiently large that some particle loss would be expected due the particles at the beam edge hitting the beam-pipe wall, and consequently, unless the alignment tolerances are tightened, steering is needed to avoid this beam loss.

The most surprising aspect of Fig 1 is how small the final betatron amplitudes are. While it was found in Ref. 3 that accumulation of centroid errors during the first lap is 

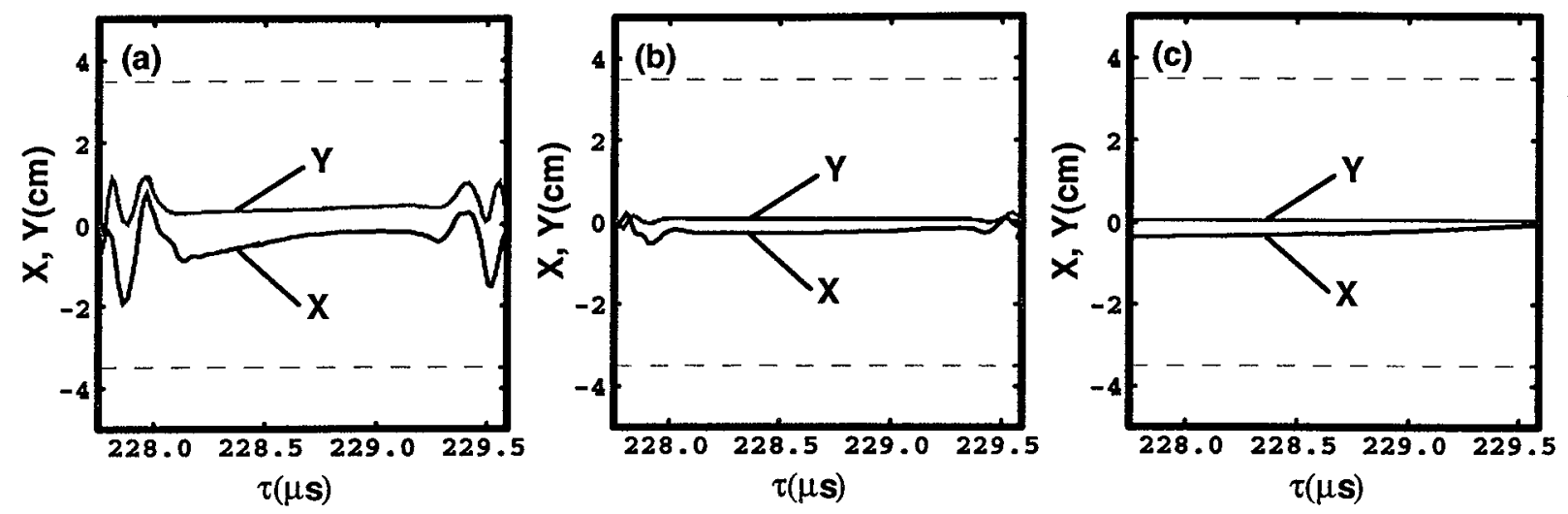

Fig. 2 Final "snapshots" of beam-centroid displacements with standard lattice errors using (a) no steering, (b) time-independent, and (c) time-dependent steering.

well modeled by a random walk, with $\langle X\rangle_{r m s}$ and $\langle Y\rangle_{r m s}$ increasing like $s^{1 / 2}$, the increase in transverse displacement over multiple laps is found to be substantially less. After roughly one lap, the envelope of maximum $X$ and $Y$ excursions for a large ensemble of cases with different errors is seen to flatten with increasing $s$ and to increase only by $15 \%$ during the remaining laps. This behavior is probably due to the periodic nature of the errors. In the absence of acceleration and compression, the errors are exactly the same on every lap, so Floquet's theorem then guarantees the existence of exactly periodic trajectories. When CIRCE is run in this constant-current mode, we indeed find that the calculated $X$ and $Y$ trajectories are each periodic with periods that are integer multiples of the recirculator circumference $s_{\max }$. The $X$ and $Y$ periods are, in general, different and vary sensitively with the quadrupole focusing strength. When the beam is accelerated or compressed sufficiently slowly, it appears that the beam is able to stay on adiabatically changing quasi-periodic orbits. The observed increase in betatron amplitudes with $s$ is perhaps due to the increasing magnitude of dipole strength errors, which are taken to be a fixed fraction of the nominal field.

\subsection{Dynamics with steering}

To demonstrate the time-dependent and time-independent steering algorithms, we use a lattice with four equally spaced steering modules. The three cases shown in Fig. 2 have the same errors but differ in the type of steering used. The snapshot in Fig. $2 b$ is obtained by applying time-independent steering, while Fig. 2c shows the corresponding snapshot when time-dependent steering is used. For comparison, Fig. 2a shows the corresponding uncorrected beam. Both cases with steering are visibly better than the uncorrected case and differ mainly in the larger ripple amplitude near the beam ends in Fig. 2b. This ripple is largely removed by time-dependent steering, but it is already sufficiently small with time-independent steering that the added cost of a time-dependent correction seems unwarranted in this experiment. 
The head-to-tail variation of $X$ seen in both Figs. 2b and 2c results from the velocity variation imposed on the beam to compress it. A simple calculation shows that the nominal displacement of a beam slice is related to the momentum error $\Delta p / p$ by

$$
X \approx \frac{1}{\rho} \frac{4 L^{2}}{\sigma_{0}^{2}} \frac{\Delta p}{p} .
$$

Here, $L$ is the half-lattice period, and $\sigma_{0}$ is the single-particle phase advance in the absence of space charge. For the case shown in Fig. 2, the head-to-tail variation in the longitudinal velocity after 15 laps is about $0.6 \%$ and the final $\sigma_{0}$ is about $33^{\circ}$, so Eq. (1) correctly predicts a head-to-tail change in $X$ of about $0.2 \mathrm{~cm}$. However, the nominal dipole voltages in this case were set so that $\Delta p / p=0$ at the beam midpoint, so that $X$ should also be zero there. Instead, the $X$ curves in Figs. $2 \mathrm{~b}$ and $2 \mathrm{c}$ are offset by about $-0.1 \mathrm{~cm}$ due to the lattice errors within the module, which introduce additional dipole fields that the algorithm interprets incorrectly as a nonzero $\Delta p / p$. This sensitivity to lattice errors within steering modules is unavoidable when any sort of beam-dynamics model is used in calculating the steering corrections. Furthermore, the use of redundant monitors to estimate the errors is unlikely to be effective because lattice errors occur between any additional monitors.

The steering algorithm is found to be rather insensitive to the order of monitoring and steering elements. Using four steering modules with an MSSMM configuration, for example, the results for either time-dependent or time-independent steering are indistinguishable from the MSMSM cases in Fig. 2. Decreasing the monitor separation to less than a quarter lattice period does not resolve enough of a betatron orbit to allow an accurate calculation of $\Delta p / p$, and increasing it to greater than half of a betatron period introduces the likelihood of aliasing. Between these extremes, little difference is seen in steering accuracy, although, as expected, higher correction voltagés are needed when steering dipoles are closer together. In addition, we find that realistic monitor errors have little effect on steering. The measured resolution of the c-probes is about $\pm 100 \mu \mathrm{m}$, but steering results from CIRCE are not visibly altered when random errors with an rms value up to $\pm 500 \mu \mathrm{m}$ are added to monitor readings.

\subsection{Spacing of steering modules}

For time-dependent steering, the maximum spacing between modules is set by one of two frequency constraints on the steering signals. The circuitry driving the steering dipoles has a cutoff frequency above which the response is nonlinear. This cutoff, which depends on the circuit design and layout and on the inductance and capacitances of the dipoles themselves, is expected to be around $30 \mathrm{MHz}$ for the LLNL small recirculator. A second frequency constraint comes from the transit time of a beam through the steering dipoles. For the beam to respond to the steering signal, the signal phase should change by much less than $2 \pi$ while a beam slice is traversing the dipole, which requires that the frequency be small compared with $\beta c / L_{d}$, where $L_{d}$ is the dipole length. For the small recirculator, with $L_{d} \approx 0.1 \mathrm{~m}$, this constraint allows a maximum frequency of about 600 $\mathrm{kHz}$ and is clearly the factor that limits the steering frequency response in the LLNL experiments. These frequency constraints limit the modules spacing because the betatronphase difference along the beam transforms a uniform displacement from the matched $X$ 

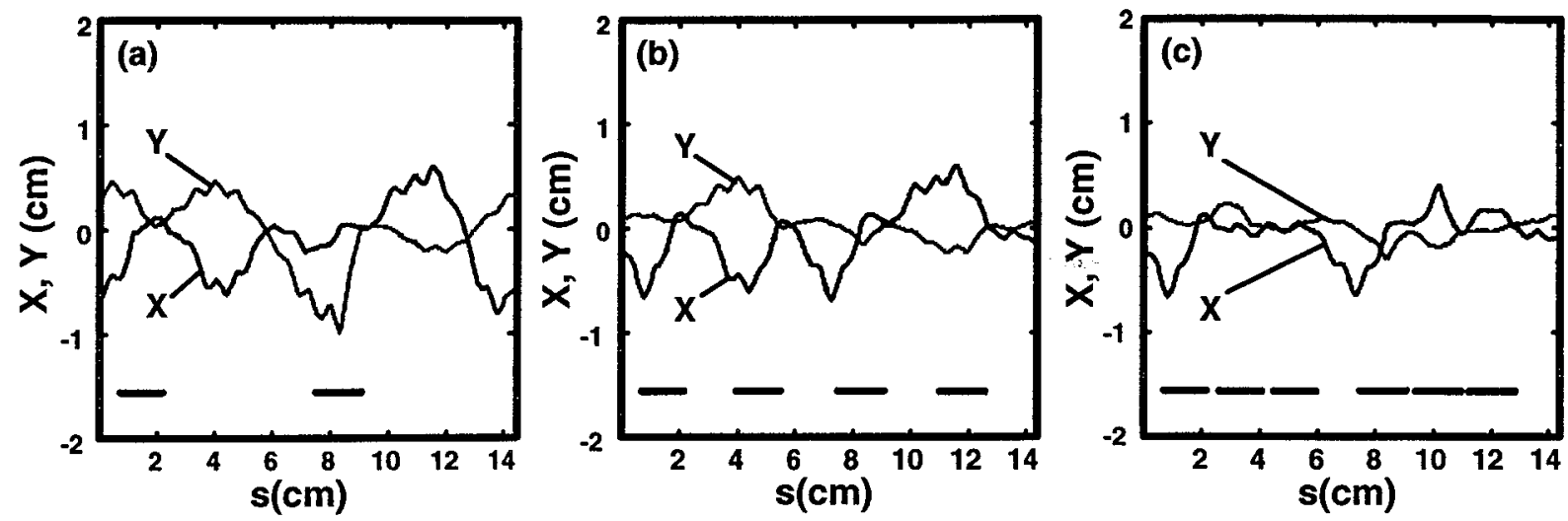

Fig. 3 Final-lap centroid "histories" with standard lattice errors using (a) two, (b) four, and (b) six time-independent steering modules. Horizontal bars indicate locations of modules.

location into centroid ripples of progressively higher frequency. For a cutoff frequency $f_{m a x}$ and a beam rise or fall time $\Delta \tau$, the maximum spacing of time-dependent steering modules is

$$
\Delta s_{\max } \approx 2 \pi f_{\max } \Delta \tau\left(\frac{\sigma_{0} R^{2}}{L K_{\max }}\right),
$$

where $K_{\max }$ is the perveance at the beam center. For the recirculator parameters in Table 1 and a cutoff frequency of $600 \mathrm{kHz}$, this expression' gives $\Delta s_{\max } \approx 45 \mathrm{~m}$. Since this spacing is larger than the circumference of the small recirculator, a single time-dependent steering module would be adequate.

The spacing of time-independent steering modules is likewise constrained by the betatron-frequency difference along the beam. Since the same kick is administered to all beam slices, the phase shift between the center and the ends that accumulates between modules must be less than about $\pi / 8$ for the direction and magnitude of the kicks to be approximately correct. Obviously, a tighter constraint on this allowed phase shift would leader to more accurate steering of the ends, but it would also reduce the allowable spacing between modules. An upper bound on module spacing, using this $\pi / 8$ phase shift, is given by

$$
\Delta s_{\max } \approx \frac{\pi}{8}\left(\frac{\sigma_{0} R^{2}}{L K_{\max }}\right),
$$

which gives $\Delta s_{\max } \approx 4 \mathrm{~m}$ for the parameters in Table 1, so that four time-independent steering modules would be needed for the experiment.

Effects of the number and spacing of steering modules are illustrated in Fig. 3. The plots show histories of the mid-pulse centroid displacement during the fifteenth lap for the standard lattice errors and two, four, and six steering modules, the positions of which 
are indicated by the bars near the bottom edge of each plot. For each case, the steering is "successful" in the sense that the beam centroid is returned approximately to the beam-pipe axis at the end of each steering module. Also, the rms average displacement in each direction decreases roughly in proportion to the module spacing. However, the maximum displacements, which occur in the two insertion/extraction (i/e) sections, are scarcely affected, and consequently, the maximum steering-dipole strengths, occurring in the modules just following the i/e sections, are not significantly reduced. Although the maximum centroid excursions for this experiment are predicted to be acceptably small, the maximum steering voltages are found to be close to the breakdown voltages expected for the c-probes and may prove to be a problem. While it is not possible to put c-probes in these sections due to the complicated geometry, steering in the plane of the ring could be done by iteratively adjusting the strength of bend dipole in the i/e sections to minimize $X$ at the next two c-probes. Tightening alignment tolerances for elements in the $\mathrm{i} / \mathrm{e}$ sections might also reduce excursions somewhat, but the largest error is expected to arise from dipole-strength errors, due to precision limits on electronics parts.

\subsection{Steering refinements}

The main weakness of the $X$ and $Y$ steering algorithms examined here is their reliance on idealized transport matrices to estimate beam dynamics within steering modules. While this transport model is tolerably accurate in the absence of lattice errors, such errors affect the beam position downstream and lead to errors in the estimated initial $X^{\prime}, Y^{\prime}$, and $\Delta p / p$, as well as in the calculation of the matched $X$ and $X^{\prime}$.

Several variants of the basic steering algorithm are being considered to reduce the effects of lattice errors within modules. A more accurate estimate of $\Delta p / p$ at the beam midpoint can be obtained by estimating the beam time-of-flight between monitors. Since the monitor signals can be used to measure the beam current $I_{b}$ as a function of time, the beam midpoint $\tau_{1 / 2}$ can be defined by

$$
\int_{-\infty}^{\tau_{1 / 2}} d \tau I_{b}=\frac{1}{2} \int_{-\infty}^{\infty} d \tau I_{b}
$$

The average beam velocity between monitors is then the difference between values of $\tau_{1 / 2}$ at the monitors divided by the separation of their midpoints, and the momentum discrepancy $\Delta p / p$ can be calculated from the nominal dipole fields. With this independent calculation of $\Delta p / p$, only two monitors are required to calculate the initial $X^{\prime}$, allowing simpler steering modules, or the beam trajectory could be fit to the redundant monitor data in some statistical way. In addition, acceleration voltages downstream could be modified to remove this average momentum error.

Another way to reduce the effects of lattice errors within steering modules is to iteratively adjust the steering fields until two or preferably three monitors downstream record the desired centroid-position values. In a straight lattice section or in a recirculator with $\Delta p / p=0$, both the centroid position and transverse velocity should be zero, so monitors 

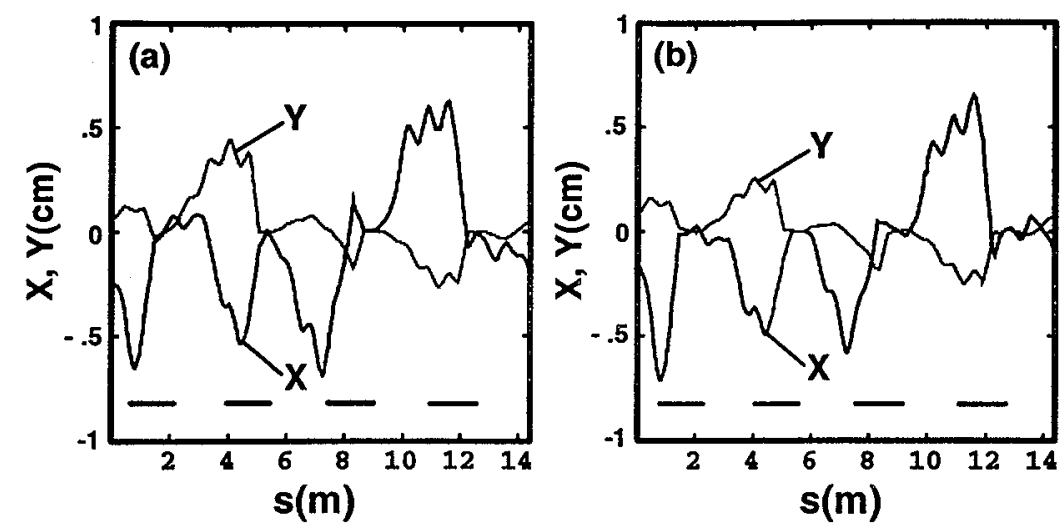

Fig. 4 Final-lap centroid "histories" with standard lattice errors using (a) basic steering algorithm and (b) iterative adjustment of steering-dipole strengths.

after the last steering dipole should give zero displacement in the absence of lattice errors. For non-zero $\Delta p / p$, the lattice transfer matrices could be used to estimate the centroid coordinates at the downstream monitors. Adding a third monitor to verify the correction is also advisable because it reduces the chance of aliasing. This method of improving steering accuracy has been tested using four steering modules in which the elements have been placed in an MSSMM arrangement. The mid-pulse centroid histories with and without iteration are shown in Fig 4. Surprisingly, the improvement is only evident in a close examination of the curves. With iteration, the centroid coordinates are seen in Fig. 4b to remain closer to the beam-pipe axis in the HLP following the second steering dipole than in the case in Fig. 4a without iteration, but for both cases, the displacements grow to nearly the same amplitude between modules. This result suggests that improving the precision of steering is not as effective in reducing average displacements as increasing the number of modules.

\section{References}

[1] J. J. Barnard, A. L. Brooks, F. Coffield, F. Deadrick, L. V. Griffith, H. C. Kirbie, V. K. Neil, M. A. Newton, A. C. Paul, L. L. Reginato, W. M. Sharp, J. Wilson, S. S. Yu, and D. L. Judd, "Study of Recirculating Induction Accelerators as Drivers for Heavy-Ion Fusion," Lawrence Livermore National Laboratory Report UCRL-LR-108095 (1992).

[2] T. C. Sangster, J. J. Barnard, V. Cianciolo, A. Friedman, D. P. Grote, E. Halaxa, R. L. Hanks, G. Kamin, H. C. Kirbie, B. G. Logan, S. M. Lund, G. Mant, W. Manning, A. W. Molvik, M. B. Nelson, W. M. Sharp, T. J. Fessenden, D. L. Judd, L. Reginato, D. Berners, H. A. Hopkins, A. Debeling, and J. Meredith, "Status of Experiments Leading to a Small Recirculator," in these proceedings.

[3] W. M. Sharp, J. J. Barnard, and S. S. Yu, "Steering Algorithms for a Heavy-Ion Recirculating Accelerator, in Proceedings of the 9th International Conference on HighPower Particle Beams, Washington, D. C., 25-29 May 1992, pp. 951-956. 
[4] F. J. Deadrick, J. J. Barnard, T. J. Fessenden, J. W. Meridith, and J. Rintamaki, "Developments of Beam Position Monitors for Heavy-Ion Recirculators," Proceedings of the 1995 Particle Accelerator Conference, Dallas, TX, 1-5 May 1995, pp. 2557-2559.

[5] W. M. Sharp, J. J. Barnard, and S. S. Yu, Part Accel. 37-38, pp. 205-213, 1992.

[6] D. P. Grote, A. Friedman, S. M. Lund, W. M Fawley, and I. Haber, "New Developments in WARP: Progress Toward End-to-End Simulation," in these proceedings. 


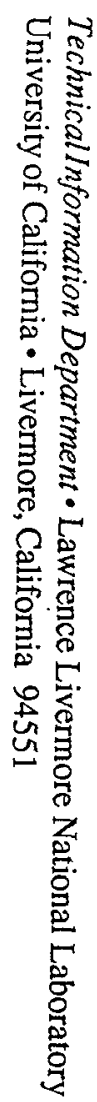

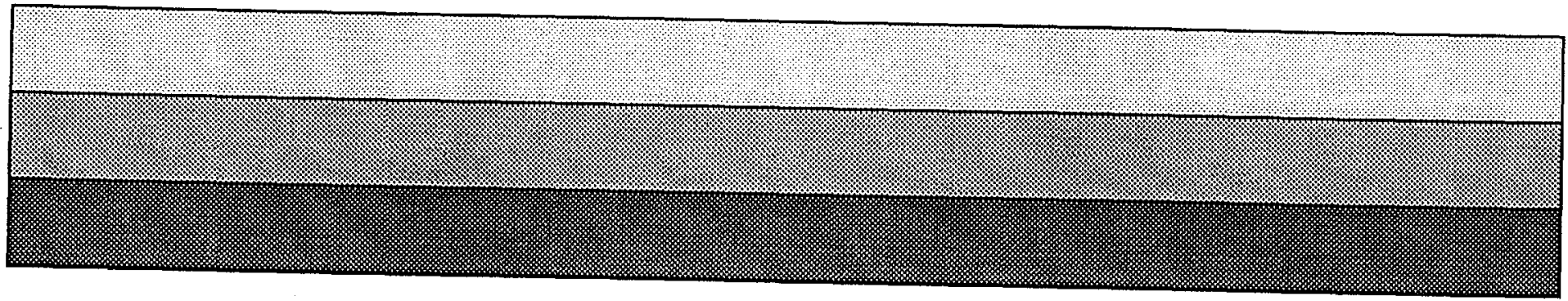

